\title{
Lo viejo en lo nuevo: la política asistencial en el Uruguay actual
}

Old wine in new bottles: social assistance policy in today's Uruguay

\section{Ximena Baráibar Ribero}

Departamento de Trabajo Social,

Facultad de Ciencias Sociales

Universidad de la República, Uruguay.

xbaraibar@gmail.com
Fecha de recepción:

18.1.21

Fecha de aceptación:

20.4.21

\section{Resumen}

Este artículo enfoca la política asistencial y el desempeño del Ministerio de Desarrollo Social en el gobierno que asumió el 1 de marzo de 2020, integrado por cinco partidos de derecha que puso fin a quince años de gestión del Frente Amplio. Con base a las propuestas de campaña electoral y declaraciones de las nuevas autoridades, el trabajo analiza la conceptualización de esa política y las medidas planteadas. Se concluye que sin perjuicio de aquellas declaraciones existe una perspectiva residual de la acción estatal; el eje de salida de la pobreza es la esperanza y la superación individual, con apoyos cercanos y teniendo las políticas sociales solamente el papel de facilitar un tránsito a oportunidades que se asumen discursivamente y no se discuten como realidad.

Palabras clave: pobreza - política asistencial Ministerio de Desarrollo Social - Frente Amplio - derecha política - Uruguay

\section{Abstract}

This article focuses on public assistance and the Ministry of Social Development performance as a new government began its rule on March 1,2020.A product of five-right wing political parties, the new administration assumed office after a 15 years rulo by following s On March 2020 a new 
government, integrated by five right wing political parties assumed government after fifteen years of Frente Amplio rule. Social assistance policy and the Ministry for Social Development role in it, were relevant issues during the electoral campaign. Based on those proposals and declarations of the new authorities, we approach the conceptualization of this policy and actions. Our main conclusion is that there is a residual perspective of state action on this matter. The way-out from poverty relies on hope and individual improvement, with close support and social policies just playing the role of enabling a transition to opportunities that are assumed and not questioned as actual reality.

Key words: poverty - assistance policy - Ministry for Social Development - Frente Amplio - political right - Uruguay.

\section{Introducción}

En octubre 2019 tuvieron lugar las elecciones nacionales en Uruguay. El Frente Amplio alcanzó el 39\% de los votos, el Partido Nacional el 28.6\%, el Partido Colorado el 12.3\% y Cabildo Abierto el 11\%. Otros tres partidos, no obtuvieron representación en la Cámara de Senadores, pero sí en la de Diputados: el Partido Ecologista Radical Intransigente con el 1.3\% de los votos, el Partido de la Gente con el 1\% y el Partido Independiente con el 0.9\% de los votos (Corte Electoral 2019). Esos resultados hicieron necesaria la realización de una segunda vuelta electoral. En ella la disputa se dio entre las fórmulas presidenciales Lacalle Pou-Argimón y Martínez-Villar. La primera obtuvo el 48.8\% del total de los votos emitidos, mientras que la segunda alcanzó el 47.3\% (Corte Electoral, 2019). Se trató de la segunda vuelta más competitiva desde 1991 (Rosenblatt et al 2019).

El triunfo de la primera fórmula -que asumió el gobierno el 1 de marzo 2020-, fue producto de un acuerdo entre los partidos Nacional, Colorado, Cabildo Abierto, de la Gente e Independiente. Esto dio lugar a lo que ese conjunto de actores políticos ha denominado "Coalición Multicolor", con una orientación de derecha y centro derecha (Rosenblatt et al 2019).Agrega Queirolo (2020) que Cabildo Abierto se ubica cerca de la extrema derecha y su participación en el gobierno acentúa la imagen del giro a la derecha en Uruguay.

Ese triunfo supuso también el cambio de fuerza política gobernante, finalizando el ciclo de quince años del Frente Amplio. Una de las acciones desarrolladas por este fue la ampliación, 
consolidación e institucionalización de la política asistencial. En 2005 creó el Ministerio de Desarrollo Social (MIDES) y puso en marcha el Plan Nacional de Atención a la Emergencia Social y luego el Plan de Equidad y la Reforma Social. En estos, las transferencias monetarias condicionadas tuvieron centralidad dentro del conjunto de programas implementados.

Uno de los asuntos que hizo parte de la campaña electoral, refirió a la política asistencial. En el proceso de negociación con los demás partidos, Luis Lacalle Pou indicó que serían tres los ministerios que estarían a cargo de personas de su confianza, siendo uno de ellos el MIDES. ${ }^{1}$ También mostraron interés en esta cartera los máximos dirigentes del Partido Colorado y de Cabildo Abierto. ${ }^{2}$ Más allá de esto, el MIDES es el único en que las tres principales autoridades (Ministro, Subsecretario y Secretario General) están a cargo de personas del partido Nacional de confianza del presidente de la República Lacalle Pou. Adicionalmente, la gestión anterior en este organismo ha sido cuestionada en varias oportunidades por las nuevas autoridades.

A partir de esta realidad este artículo tiene como objetivo analizar la manera en que la política asistencial es conceptualizada por el Partido Nacional y la Coalición Multicolor. Dada la centralidad que tiene el mencionado partido en las definiciones y orientaciones del MIDES, se analizan sus propuestas en la campaña electoral, el documento de acuerdo entre los cinco partidos de la coalición triunfante, así como declaraciones realizadas por el Ministro de esa cartera Pablo Bartol y el Presidente Luis Lacalle Pou.

El análisis de los programas permite conocer las orientaciones y propuestas que habrán de guiar la acción del gobierno uruguayo hasta 2025, en un tema de alta sensibilidad y relevancia, dada la cantidad de población que accede a niveles de protección social a partir de la política asistencial, pero, además, en términos de la mirada que contribuyen a conformar sobre la realidad. Como indica Grassi (2018 b) en los principios que fundamentan las políticas y en las interpretaciones y argumentos, es posible reconocer qué sociedad aflora como presupuesta y como deseable y consecuentemente qué Estado se configura y como enlaza a sus miembros.

Se concluye que hay una perspectiva residual de la acción del Estado, concebido como una pesada máquina burocrática y generador de dependencia. La idea de autonomía -en la que se insiste- es entendida en oposición a la intervención pública. El eje central de salida de la pobreza es la esperanza, sin ninguna vinculación con la desigualdad. La apuesta es a la superación individual, con apoyos del entorno cercano (familia, barrio), siendo el papel central de las políticas sociales, favorecer ese tránsito. Uruguay y más ampliamente América Latina, ya ha conocido estas miradas, generando la agudización de la pobreza y desigualdad como resultado y drama social.

La aparición en Uruguay de la pandemia se reconoció pocos días después de inaugurado el nuevo gobierno el 1 de marzo 2020. Sin embargo las acciones emprendidas a su 
respecto no llevaron a las autoridades públicas a un reenfoque de su política asistencial, que constituye el objeto de este texto. Pese a la situación de emergencia las orientaciones de política de las acciones emprendidas se disciernen con facilidad: aumentos mínimos de las transferencias de ingresos ya existentes, sin ampliación de cobertura; apoyos alimentarios, también de muy bajo monto y sin que -fuera de las indicaciones sobre quienes pueden postular- sean claros los mecanismos de adjudicación. Finalmente, en relación a las ollas populares -una de las expresiones más dramáticas de la crisis- que emergen de manera casi inmediata con la pandemia, la actitud del gobierno ha sido variada. Pese a alejarse de las recomendaciones sanitarias, han sido valoradas como expresiones solidarias, lo que básicamente ha supuesto el traslado a las redes comunitarias de la respuesta al hambre y sin interpelar al Estado sobre su ausencia. Los apoyos a las mismas -pese a reiterarse como anuncios- han sido magros y tardíos y han sido sospechadas por autoridades por su carácter político, sin asumir que el hambre, es un hecho de esa naturaleza. La ley de presupuesto sancionada en agosto 2020 para el quinquenio 2020-2024 reitera en lo fundamental los planteamientos formulados por el Partido Nacional. Estos aspectos quedan más allá del objeto del presente artículo y remiten a un estudio ulterior.

El artículo se organiza en cuatro partes. En la primera se conceptualiza la política asistencial y en la siguiente, se presentan los regímenes de bienestar de América Latina. Luego se analizan las orientaciones del Partido Nacional y lo planteado por la coalición multicolor; el artículo se cierra con un apartado de síntesis y conclusiones.

\section{Qué se entiende por política asistencial}

La política asistencial ha sido conceptualizada básicamente de dos formas, que no son contrapuestas sino que presentan énfasis particulares: como acciones dirigidas a los pobres y como prestaciones no contributivas.

En el primer caso, se encuentra la formulación de Andrenacci y Repetto (2006), para quienes:

La política asistencial es un conjunto de mecanismos de transferencia de bienes y servicios a los 'pobres' en sentido contemporáneo: aquellos individuos y grupos que presentan dificultades y riesgos específicos para resolver su reproducción a través del mercado de trabajo, o acceder a las condiciones de vida básicas garantizadas por el complejo de políticas universales

(Andrenacci y Repetto 2006: 85).

Para Campana (2014) la asistencia social constituye una modalidad privilegiada de gobierno de la pobreza y alude al conjunto de intervenciones sociales del Estado sobre aquellos 
individuos, grupos y/o poblaciones definidos como pobres, en función de una serie de atributos previamente construidos e identificados. También Arias (2012) asocia la política asistencial a acciones estatales orientadas hacia los pobres, pero reconociendo que en la condición de pobreza son otros los determinantes de mayor peso. Son aquellas intervenciones que

(...) se dirigen a los pobres como sujetos, sabiendo que si bien operan incluso de manera más determinante sobre la situación de pobreza, otro conjunto importante de dispositivos institucionales, como las regulaciones laborales, la educación pública o el sistema jubilatorio, no son operatorias dirigidas a la pobreza, aunque modifiquen de manera determinante la distribución de la riqueza en general y en especial sobre los pobres

(Arias, 2012: 39).

Otra forma de delimitar la política asistencial surge de definirla como una prestación no contributiva. Cecchini y Martínez (2011) la ubican como una de las funciones de la protección social, junto a la seguridad social y la regulación de los mercados laborales y básicamente como orientada a pobres y vulnerables. Indican que la protección social tiene por objetivo compensar la caída de ingresos de forma de mantener niveles de calidad de vida consensuados como esenciales, posibilitar el acceso a los servicios sociales garantizando el capital humano y procurar la universalización del trabajo decente.

En ese marco, el empleo es un elemento fundamental, en tanto permite acceder a ingresos estables y a la protección social contributiva. Esta conforma la seguridad social y se orienta al aseguramiento presente y futuro, procurando mantener un mínimo de calidad de vida durante la etapa activa y la inactiva. A esto se suman los seguros de salud, los beneficios asociados a la maternidad y en algunos casos, otros como las asignaciones familiares. Se trata de beneficios contributivos, aun cuando el monto de los aportes puede variar de acuerdo al estrato socioeconómico de los trabajadores y su permanencia en el mercado formal de trabajo. Presentan gran debilidad en América Latina, debido -entre otros aspectos- a la realidad de los mercados de trabajo, lo que genera, la necesidad de instrumentos de protección social no contributiva. Es frecuentemente conocida como asistencia social y hace referencia a

(...) el conjunto de programas de transferencias y subsidios públicos, normalmente financiados mediante impuestos a los ingresos generales bajo el principio de solidaridad. Sus beneficios no se relacionan con la existencia de contribuciones previa

(Cecchini y Martínez 2011: 147). 
En una perspectiva divergente, Boschetti (2001) considera que es el trabajo el elemento que asegura la inclusión en la previsión, definiendo la naturaleza y el monto de los derechos existentes. A la inversa, las prestaciones de renta aseguradas por la asistencia están destinadas a los que, por algún tipo de incapacidad (edad y/o deficiencia) están imposibilitados de trabajar. Entiende que el seguro y la asistencia constituyen una unidad de contrarios, imponiéndose uno u otro según las relaciones económicas, sociales y políticas de cada país. Finaliza indicando que más allá de esa definición, ambas lógicas se dirigen a amparar aspectos de un mismo fenómeno: la relación con el trabajo. A pesar de no depender de las contribuciones vinculadas a la seguridad social, la autora problematiza el carácter no contributivo, a partir de su concepción sobre la asistencia social en una sociedad desigual.

Un segundo aspecto relevante es que la política asistencial es entendida como uno de los campos de las políticas sociales. Estas son concebidas como

(...) todas aquellas intervenciones públicas que regulan las formas en que la población se reproduce y socializa (sobrevive físicamente $y$ se inserta en el mundo del trabajo y en el espacio sociocultural del Estado-nación) y que protegen a la población de situaciones que ponen en riesgo esos procesos (Soldano y Andrenacci, 2005: 32).

Agregan los autores que las políticas sociales se expresan en la política de trabajo, la de servicios universales y la asistencial y tienen clivajes temporales y espaciales. Esto supone, que, aunque siempre presentes, sus énfasis varían entre los países y tiempos históricos. Por su parte, destaca Grassi (2018 b) la centralidad que las políticas sociales tienen en la determinación de las condiciones de vida colectiva (de toda la población y no solo de los pobres), en tanto son parte del andamiaje estatal que sostiene la vida social, el ser individuo y su posibilidad de participar y auto valerse.

Agrega que la política social puede tender a consolidar el patrimonio social que de seguridad a la vida de todos, como a ponerlo en riesgo y sostener distancias sociales. Esto ocurre cuando la protección de la vida se hace depender prioritariamente de la capacidad privada para el aseguramiento individual, reservándose la eventual intervención estatal para poblaciones vulnerables. En este caso, las protecciones, conllevan el estigma de la flaqueza o la inutilidad y no disponen de ninguna seguridad, llevando la existencia en condiciones precarias (Grassi, 2018 b).

Otro aspecto a considerar es que la política social -y, por tanto, la asistencial- además de asuntos técnicos, supone centralmente, aspectos políticos. Esto implica que la expresión que porta es siempre objeto de disputa y remite a una forma de comprensión de la 
cuestión social. Entiende Grassi (2018 a; 2018 b) que las preguntas por el Estado y las instituciones que son soportes de la vida social se inscriben en disputas por la imposición de alguna visión del mundo. Agrega que el Estado tiene capacidad de normar y normalizar, en tanto se constituye en un actor y ámbito en la producción de los problemas sociales, en la delimitación de su propia responsabilidad, en la definición de sujetos merecedores de sus intervenciones y de las condiciones para dicho merecimiento. De esta forma: "Los medios y recursos de producción de visiones del mundo son, también, los medios y recursos de producción del mundo” (Grassi, 2018 a: 59).

Finalmente, se considera que el análisis de la política asistencial adquiere mayor potencia cuando trasciende la sola mirada a la misma y se pone en relación con otras esferas de provisión de bienestar. Midaglia y Silveira (2011) señalan que el papel que las propuestas pueden desempeñar en la protección, no depende solo de sí mismas, sino que guarda relación con el contexto socioeconómico y el marco político-institucional de bienestar en el que se inscriben.

\section{Los regímenes de bienestar en América Latina.}

En el análisis sobre el acceso al bienestar, es Esping-Andersen (2000) un autor clásico en la materia. Hay cuatro aspectos a destacar dentro de su producción. El primero surge de la propia idea de regímenes de bienestar, que supone que, en la producción y distribución de bienestar, es preciso considerar tres esferas: el mercado, el Estado y también la familia. Asimismo, puede ser considerado el tercer sector, donde incluye las organizaciones voluntarias o sin ánimo de lucro.

El segundo aspecto es que analizar las formas concretas de los regímenes de bienestar, es importante a nivel general (en tanto lo que ocurra con y en cualquiera de los tres componentes, está relacionado con lo que suceda con los otros dos), pero también a nivel individual. Más allá de las orientaciones generales, dirá que a nivel micro, el bienestar de los individuos dependerá de como los individuos agrupen los recursos y posibilidades de cada una de estas esferas.

Luego, aunque presentes siempre los tres pilares mencionados, toman formas distintas. En esta diferenciación es central el papel del Estado, permitiendo una mayor o menor desmercantilización y desfamiliarización, con impactos en la estratificación social. El primer concepto aspira a captar el grado en el que los estados de bienestar debilitan el nexo monetario al garantizar derechos independientes de la participación en el mercado. De manera similar, la desfamiliarización supone disponibilidad de recursos, por parte del individuo, de manera independiente a las reciprocidades familiares o conyugales (Esping-Andersen, 2000).

Finalmente, considera que, en los países desarrollados, la presencia diversa de estos tres pilares da lugar a tres regímenes de bienestar. El liberal expresa un compromiso político 
por minimizar el Estado, individualizar los riesgos y fomentar las soluciones de mercado y así desfavorecer los derechos ciudadanos. De igual manera que en los regímenes conservadores, también aquí son residuales los servicios familiares, aunque por distintas razones. Los liberales los entienden como una actividad del mercado y como una responsabilidad individual. Por su parte, el régimen socialdemócrata se caracteriza por el universalismo y la socialización global de los riesgos (siendo marginales los servicios de bienestar privados), generosos niveles de subsidio, y el igualitarismo. También es central en este régimen su fuerte acento en la desfamilización. Finalmente, el régimen conservador se caracteriza por la segmentación de estatus y familiarismo. Esto supone la protección social sesgada a favor del varón cabeza de familia y esta como responsable central del bienestar de sus miembros. Es marginal la provisión de bienestar por parte del mercado privado, como consecuencia de la seguridad social obligatoria, complementada con planes de pensiones ad hoc y más o menos residuales para aquellos que están fuera de una relación laboral 'normal'. 3

Con estas referencias, distintos autores y autoras han analizado el bienestar en América Latina. Aquí y de acuerdo a Ubasart-González y Minteguiaga (2017), es en la década de 1980 que se encuentran los primeros esfuerzos por calificar los modelos de Estados sociales y diez años después, por analizar la realidad en clave de regímenes de bienestar. Como se verá, existe coincidencia en tres aspectos, siendo el primero, la necesidad y conveniencia de analizar el acceso al bienestar y sus fuentes, también en el continente. Asimismo, es compartida la distancia visualizada entre este último y los países desarrollados, y también -y más allá de los trazos generales- la diversidad entre los países latinoamericanos. Centralmente se destacan las diferencias en los mercados laborales (y las protecciones asociadas) y en el papel del Estado, lo que lleva a que tengan las familias una relevancia mayor en el acceso al bienestar y la protección social.

Barba Solano (2019) entiende que es preciso usar de manera crítica el concepto de regímenes de bienestar en el mundo en desarrollo. Más allá de esto, destaca la importancia de las políticas e instituciones sociales para desmercantilizar o desfamiliarizar los riesgos sociales, siendo fundamentales para la distribución del poder en cada sociedad y para respaldar o modificar la estructura social desigual que genera el mercado -particularmente el laboral-, así como la división de tareas dentro de la familia. Para el autor, la mirada crítica es precisa en tanto el trabajo no es asalariado y los mercados son imperfectos. Asimismo, las comunidades son clientelistas y patriarcales los hogares. Finalmente, de manera frecuente, el Estado es débil, siendo uno más entre otros ámbitos del bienestar o es penetrado por otros poderes fácticos (Barba Solano, 2019). Por su parte, para Ubasart-González y Minteguiaga (2017) los regímenes de bienestar en América Latina, no tuvieron presencia central del Estado, lo cual no supone dejar de analizarlo, dado que por acción u omisión participa en la extracción de recursos y su distribución y define la regulación de los mercados, en particular el laboral. 
Lo señalado plantea para el autor, la necesidad de proponer otros tipos de regímenes de bienestar, identificando en la región y entre 1940 y 1980, tres de estos: universalistas, duales y excluyentes. Los primeros alcanzaron mayores niveles en el proceso de salarización, junto a un importante despliegue de instituciones y políticas que garantizaron, para amplios sectores de la sociedad, derechos sociales. Los últimos, no desarrollaron sistemas de bienestar y de esta forma, concentraron la protección social en una pequeña oligarquía y excluyeron a la mayoría de la población. Finalmente, los duales, siguieron los patrones de los universalistas en las zonas urbanas, pero excluyeron a la población del medio rural (Barba Solano, 2019).

El primer conjunto -en el que incluye a Argentina, Chile, Costa Rica y Uruguay- alcanzó los niveles más altos de protección pública y de ampliación de la ciudadanía social. Ubica en el tercero a Honduras, Nicaragua, Guatemala, El Salvador, Bolivia, Ecuador, Paraguay y Perú. Aquí, los recursos de los pobres para hacer frente a los riesgos sociales fueron sus familias y redes comunitarias. Brasil y México integran los regímenes duales (Barba Solano 2018).

Juliana Martínez Franzoni, catedrática de la Universidad de Costa Rica, realiza un esfuerzo importante para estudiar el bienestar en América Latina, fundamentalmente después de los años 80 y 90 del siglo pasado. Lo concibe como una capacidad para hacerle frente a los riesgos y define a los regímenes de bienestar como la "constelación de prácticas, normas y discursos relativos al 'que' y a 'quienes' les corresponde la producción de dicho bienestar"; involucran prácticas de asignación de recursos y percepciones, discursos y orientaciones normativas (Martínez Franzoni 2008: 5).

Comparte las tres esferas planteadas por Esping Andersen (2000), pero avanza en problematizar lo que ocurre y supone la familia. Dirá la autora que es a través de las relaciones interpersonales, del intercambio mercantil, de los mecanismos comunitarios o estatales, que cada colectivo lidiará con los riesgos asociados al estar vivos. En las sociedades capitalistas, el intercambio mercantil es el principal mecanismo de asignación de recursos, lo que supone que el acceso a bienes y servicios depende de manera fundamental de los ingresos que las personas generen. Agrega que no es el único mecanismo de asignación de recursos -y para muchas personas ni siquiera el principal-; esto también ocurre a través del Estado y del trabajo no remunerado, especialmente el femenino, dentro de la familia (Martínez Franzoni 2008).

Entiende que los regímenes de bienestar no constituyen interrelaciones armónicas y sinérgicas, y, al contrario, están atravesados por tensiones dado que las prácticas de asignación de recursos están basadas en relaciones de poder. Además, esas tensiones serán mayores cuanto más débiles sean las prácticas de asignación de recursos o más ‘fallas' tengan unas u otras. En América Latina los mercados laborales generan empleo insuficiente y precario. Junto a la crisis en el empleo formal, destaca la economía de trabajos precarios e informales y el desempleo estructural. Asimismo, la presencia del Estado es débil en muchos países; es reducida la inversión social y también los recursos públicos destinados a la protección social. 
Ante mercados y Estados que fallan en la asignación de recursos suficientes, la autora se pregunta a qué recurrir, si no es a los vínculos más cercanos. Esto hace que, para amplios sectores de la población latinoamericana, básicamente aquellos librados a su suerte, tenga gran relevancia la familia, intentando compensar las 'fallas' institucionales. Esto lleva a 'informalizar' en mayor grado la producción de bienestar (Martínez Franzoni 2008).

La autora continúa e indica que la familia, supone trabajo no remunerado y este es especialmente el femenino. Esto hace que en la consideración de la estructura de los riesgos con la que lidian las personas, además de la estratificación económica, considere la estratificación de género. Esta dimensión es incorporada de manera central en el análisis de los regímenes de bienestar, dado que, en torno a la división sexual del trabajo, se organizan las prácticas de asignación de los recursos (Martínez Franzoni 2008).

A partir de estas consideraciones, Martínez Franzoni identifica tres tipos de regímenes de bienestar. En el primero, o estatal-productivista, ubica a Argentina y Chile. Brasil, Costa Rica, México, Panamá u Uruguay forman parte del conglomerado estatal-proteccionista, mientras que Colombia, Ecuador, El Salvador, Guatemala, Perú, República Dominicana,Venezuela, Bolivia, Honduras, Nicaragua y Paraguay, integran el informal-familiarista. Los dos primeros se distinguen en grado en la mercantilización y la familiarización, y tienen diferencias cualitativas en la desmercantilización. Ambos presentan diferencias importantes con el tercer conglomerado tanto de grado (por ejemplo, en la efectividad de los mercados laborales para absorber la fuerza de trabajo) como cualitativas (por ejemplo, en que sean expulsores o atrayentes de la población trabajadora). En este, los países comparten menores grados de desmercantilización de la fuerza de trabajo, escasos grados de dsemercantilización y altos grados de familiarización del bienestar (Martínez Franzoni 2008).

En general los países en los conglomerados primero y segundo tienen mayores capacidades para absorber la fuerza de trabajo de manera asalariada. La principal diferencia tiene que ver con el ingreso anual por persona. En el tercer conglomerado la población recurre en mayor proporción al autoempleo y a los mercados laborales transnacionales. Pese a lo señalado, los dos primeros conglomerados también proveen insuficiente empleo formal, debiendo muchas personas, recurrir al autoempleo. Entonces, a pesar de existir diferencias considerables entre conglomerados, los tres tienen tendencia a la informalización en la mercantilización de la fuerza de trabajo (Martínez Franzoni 2008).

La desmercantilización del bienestar es mayor en los conglomerados 1 y 2; salvo en educación y seguridad social, es mayor en el 1 que en el 2. En este último parece observarse más relevancia de la protección social organizada colectivamente y estratificada según las ocupaciones. En cambio, mayor presencia de la protección social individual es lo que observa en el primer conglomerado. Al compararlo con los conglomerados 1 y 2, la desmercantilización en el tercero, es muy baja (op.cit). 
Lo señalado llevará a la existencia de altos grados de familización en los tres conglomerados. En los dos primeros, son las familias nucleares las que tienen un papel más importante, mientras que esto ocurre con las extensas y compuestas en el tercer conjunto de países. Como síntesis, considera que:

(...) todos los regímenes latinoamericanos son, en alguna medida, informales, en tanto una proporción de la población no puede, en ninguno de los tres conglomerados, lidiar razonablemente con los riesgos sociales a partir de las lógicas claramente diferenciadas entre el intercambio mercantil, las relaciones familiares y la política pública. (...) Por lo tanto, una proporción variable de la población, en alguna medida, depende de los arreglos familiares $y$ comunitarios para asumir las prácticas cuyas lógicas corresponden a los mercados, en particular laborales, o a los Estados locales o nacionales

(Martínez,Franzoni 2008: 113).

Los arreglos diversos tienen impactos en las condiciones de vida, destacando una mayor efectividad de los regímenes estatales que del familiarista. Tomando la mortalidad infantil, la esperanza de vida y la esperanza de vida escolar como indicadores de la estructura de riesgos, observa la autora diferencias marcadas entre los regímenes.

Esa realidad experimentó algunas modificaciones a partir de los gobiernos progresistas que desde inicios del presente siglo buscaron revertir con desigual éxito las condiciones materiales y simbólicas de vida de la etapa anterior: intervenciones en el mercado de empleo, relativas a regulaciones laborales, la formalización de la fuerza de trabajo y aumentos del salario mínimo (Ubasart-González y Minteguiaga 2017; Midaglia y Castillo 2018). Como respuesta al dramático panorama producto de los procesos de ajuste estructural y reforma, la política asistencial tomó primero la forma de los Fondos de Inversión Social (FIS) y sobre finales del siglo pasado y fundamentalmente en el actual, ampliando su desarrollo particularmente con los programas de transferencias condicionadas (PTC) y con la creación de una institucionalidad específica como son los Ministerios de Desarrollo Social.

Estas modificaciones se llevaron a cabo, sin embargo, sin cambios sustantivos en el diseño contributivo del sistema original y sus variantes mercado-céntricas y focalizadoras de la década de 1990 desarrolladas para atacar las fisuras de los regímenes contributivos. En este sentido Martínez Franzoni y Sánchez-Ancochea (2016:217-218) concluyen:

(...) el acceso contributivo sigue siendo un rasgo fundamental de todos los regímenes de bienestar latinoamericanos. 
Las considerables mejoras en materia de cobertura se combinan con persistentes brechas en materia de suficiencia $y$ equidad según se trate de programas contributivo y no contributivos. (...) Las transformaciones recientes han sido, sin duda, positivas desde el punto de vista de la cobertura, pero negativas para corregir la inequidad y muy variables entre los países respecto a la suficiencia de las prestaciones. El resultado es la permanencia de un régimen de bienestar que -aunque con profundas variaciones interregionalespromueve una incorporación social altamente segmentada.

Finalmente, interesa mencionar el impacto que estas modificaciones producen en la configuración del individuo, de acuerdo a lo planteado por Merklen (2013). Este autor considera que, durante el desarrollo del que denomina Estado Social, se entendía que el riesgo refería a las contingencias que las diferentes actividades sociales o económicas acarreaban sobre los individuos. La sociedad era responsable de las consecuencias negativas de la actividad económica sobre el trabajador y, por tanto, debía protegerlo a él y a las familias. Al individuo, afirma, lo resguardaba la sociedad. Este estado procuró institucionalizar regulaciones sociales y protecciones, buscando la producción de un orden social del que surgían márgenes de libertad e independencia para el conjunto de los individuos. Esa realidad cambia a partir de la década de 1980, cuando el riesgo es invocado para indicar el peligro que, por sus actividades o su falta de precaución, los individuos hacen correr a la comunidad o, más precisamente, a otros individuos. Se tratará de ser responsable de sí mismo y ser activo con el fin de minimizar eventuales costos en la comunidad: una concepción liberal del individuo.

Sigue indicando el autor que, en esta orientación, la libertad es presentada de forma esencialmente negativa y asociada al relajamiento de las restricciones y el proceso de individuación como el 'liberar al individuo'. La sociedad es concebida como límite o imposición a la libertad individual y esta no puede ser pensada como resultado de un vínculo social o de una acción colectiva que permitan 'puntos de apoyo' que habiliten nuevas oportunidades de acción (Merklen, 2013).

Esta concepción también tiende a negar que los individuos son desiguales en sus aspiraciones a la individuación y en las condiciones efectivas para esto. En cambio

(...) no están igualmente provistos para asegurarse por sí mismos contra las consecuencias de su participación en la vida social y los riesgos de la existencia. (...) La exposición al riesgo es desigual, como desiguales son los recursos de los cuales dispone cada cual para protegerse de él

(Merklen, 2013: 52). 


\section{La política asistencial en el nuevo gobierno en Uruguay}

Un plan de gobierno, señala Estela Grassi, expresa una visión de país y de lo que debe considerarse un buen gobierno, $\mathrm{y}$

(...) principalmente de que protecciones corresponden a cada quién, porque y a través de que medios. Es decir, cual o como será el programa que vaya a ser reconocido $y$ aceptado como 'mejor para todos', a quienes comprende el 'todos' $y$ sobre quienes pesa rechazo o indiferencia; $y$ también, su estabilización como normalidad de la vida social (Grassi, 2018 a: 73).

La asunción del nuevo gobierno significó poner fin a quince años de gestión del Frente Amplio. Este asumió el gobierno nacional en 2005 en un contexto social complejo producto de la crisis económica del año 2002, aunque también resultado de procesos más amplios (Midaglia y Antía 2011). Desde su inicio el gobierno del Frente Amplio otorgó jerarquía a la agenda social; esto se expresó en la atención a las demandas planteadas por los sindicatos en tanto actores colectivos históricamente aliados y a la situación de los sectores sociales empobrecidos. Aunque hubo acciones orientadas directamente los pobres son de larga en Uruguay, con impulso a partir de los años noventa, fue con los gobiernos del Frente Amplio, que la política asistencial se amplió, diversificó e institucionalizó, convirtiéndose en un producto fuertemente asociado a su gestión.

En marzo 2005 se creó el Ministerio de Desarrollo Social. La ley n 17.866 , le estableció trece competencias, agrupables en cuatro esferas: la puesta en marcha del Plan de Atención a la Emergencia Social; la gestión de políticas y planes en áreas específicas; la articulación y coordinación de la oferta pública social; y la organización y gestión de un sistema de información de la oferta pública social. La política asistencial tuvo, además, tres mojones fundamentales. El primero fue el Plan Nacional de Atención a la Emergencia Social (PANES), configurado como la mayor estrategia de respuesta a la emergencia social, desarrollado entre 2005 y 2007. ${ }^{4}$ En 2008 (y sin plazo de finalización) comenzó a implementarse el Plan de Equidad (PE) institucionalizando la política asistencial al colocarla como parte permanente dentro de las prestaciones del Estado. Se integró por dos grandes componentes: los estructurales de la matriz de protección social ${ }^{5}$ y la red moderna de asistencia (RAIS) orientada a:

amparar a todos los grupos sociales y en particular a aquellos sectores socioeconómicos que tienen restringidas sus oportunidades de incorporarse al mercado de empleo por diversas razones

(Consejo Nacional de Políticas Sociales -CNPS-,2008:9) 
y es entendida como articulada con el resto del sistema de protección social.

El tercer mojón -ya en el segundo gobierno del Frente Amplio- fue la Reforma Social, aprobada en 2011. Trató de la construcción de la matriz de protección social, así como del nexo entre los componentes mencionados en el Plan de Equidad; su objetivo fue

Asegurar el pleno ejercicio de los derechos de todos los habitantes del Uruguay en condiciones de equidad, en especial aquellos que se encuentran en situaciones de vulnerabilidad social; garantizar la igualdad de oportunidades en el acceso a trabajo digno, servicios sociales universales $y$ prestaciones sociales de calidad; $y$ promover la cohesión social

(CNPS 2013:16).

Dentro de esos mojones, pero trascendiéndolos, fueron desarrollados una cantidad y variedad importante de programas (entre otros, Uruguay Trabaja, Rutas de Salida, trabajo con personas en situación de calle, Mejoramiento del Hábitat, intervenciones en salud y educación, Programas de proximidad); tuvieron centralidad de transferencias condicionadas de ingresos, los que tendrán relevancia tanto en términos de población cubierta como de recursos destinados. Esto habría de ocurrir con el Ingreso Ciudadano dentro del PANES y luego en el Plan de Equidad, modificando las Asignaciones Familiares, existentes desde 1943.

Con este enmarcamiento se desenvolvió la campaña electoral de 2019 y el inicio del de la Coalición Multicolor.

El programa de gobierno del Partido Nacional, denominado 'Lo que nos une', se organiza en cinco puntos. En uno de ellos -"La integración como tarea de todos"- se ubican centralmente los asuntos referidos al objeto de este artículo. Se trata de un documento extenso, que desarrolla los distintos componentes y sus orientaciones. Por su parte, el acuerdo -“Compromiso por el país"- firmado en noviembre 2019 por los líderes de los partidos que integran la Coalición Multicolor se compone de trece puntos, uno de ellos orientado a 'Proteger a los más débiles'. Es un documento de menor extensión que el anterior, donde se enuncian medidas casi sin desarrollar lo que ellas suponen ni sus fundamentos.

La relevancia de las orientaciones del Partido Nacional, no surge solo de ser el partido mayoritario, sino además de la configuración del MIDES. Pese a ganar la segunda vuelta electoral por acuerdo con otros partidos, y al interés expresado por estos, es el único Ministerio, cuyos tres principales funcionarios autoridades provienen de ese partido.

De acuerdo a una nota de prensa, ${ }^{6}$ el presidente Lacalle Pou conoce y admira al Ministro Pablo Bartol desde 2007, por su trabajo en el centro educativo Los Pinos ubicado en 
Casavalle, una zona pobre de Montevideo. Se trata de una iniciativa del Opus Dei, creada y dirigida por el actual Ministro desde 1998 hasta asumir ese cargo. Agrega la nota que, dada la falta de experiencia política, Lacalle Pou eligió como Subsecretario al diputado y ex intendente del departamento de Flores, Armando Castaingdebat. Este funcionario proviene de la política; el presidente Lacalle Pou entiende que toda secretaría de Estado necesita de alguien que conozca desde adentro el mundo de la política para poder negociar y llegar a acuerdos que permitan avanzar. Además, proviene del interior; la idea es trabajar en permanente contacto con los gobiernos departamentales. Finalmente, para el cargo de Director General el presidente designó a uno de sus hombres de mayor confianza política y técnica, teniendo como principal objetivo que las cuentas estén claras, limpias y en orden. Nicolás Martinelli es el encargado de mirar detenidamente cada paso que se haya dado en las administraciones anteriores y que se vaya a dar en la actual.

La mirada sobre la pobreza y la política asistencial, debe ser contextualizada en la relevancia que adopta para el nuevo gobierno la idea de libertad. Lacalle Pou ha afirmado, "Soy liberal, creo en la libertad de las personas, creo que la gente es responsable de manejar su libertad y eso sí es un cambio sustancial en el concepto del gobierno que va a empezar el 1 de marzo". ${ }^{7}$ Este fue el eje principal de su discurso al asumir la Presidencia de la República, indicando como síntesis que

Estamos convencidos que si al final del período los uruguayos somos más libres, habremos hecho bien las cosas, de lo contrario habremos fallado en lo esencial. Permítanme entonces invitarlos a trabajar por la libertad en todas sus formas (Lacalle Pou, 2020)

También fue uno de los tópicos en el discurso del Ministro Bartol (2020), al asumir su cargo en el MIDES. Señaló el nuevo ministro que el organismo tiene que brindar una "cuota de inspiración" para que las personas salgan de una situación y aprovechen las oportunidades, en el marco del respeto a la libertad individual.Agregó que sentirse libre no es sentirse solo y para ello son importantes los referentes barriales.

La preocupación central del Parrido Nacional (2019) es la integración social. Considera que existe un desajuste entre la realidad económica y la social, observando una economía que crece, pero al mismo tiempo una sociedad cada vez más fracturada. Esto se expresa, de acuerdo al documento, en los niveles de exclusión, las tasas de criminalidad, las desigualdades educativas y los problemas de vivienda. Es entendida como una situación que además de injusta, es peligrosa para el futuro. De no modificarse, el país dejará su trayectoria de país integrado y pacífico y se convertirá en una sociedad divida en dos bloques enfrentados. 
También integra las preocupaciones de la Coalición Multicolor. Parte de destacar que el Uruguay siempre fue la sociedad más integrada y con mejor distribución del ingreso en América Latina. Estos partidos políticos consideran que, en la actualidad, el país se enfrenta a la fractura social, la marginalidad y la vulnerabilidad económica de muchos hogares. En función de esto, entienden como uno de los principales desafíos del país, el recuperar la integración y la justicia social, rompiendo los ciclos que generan desigualdad y exclusión (VVAA, 2019).

Este último documento no avanza en explicar los motivos que llevan a los procesos destacados. En el caso del Partido Nacional se trata de un fracaso que se considera se expresa en fractura social. Además, y como segundo aspecto relevante, la política asistencial no aparece entendida como soporte, como dispositivo que tienda (mínimamente) a reducir la inseguridad social. La mirada es -y se reiterará-, en clave de dependencia del Estado:

Si bien se redujo la pobreza monetaria, eso es el resultado de transferencias económicas realizadas desde el gobierno. En demasiados casos, esa mejora en los ingresos de hogares vulnerables no ha ido acompañada del fortalecimiento de capacidades que permitan sostenerse en forma autónoma. No estamos, por tanto, ante un proceso de mejora sostenible sino ante una situación de dependencia que queda ligada al estado de las cuentas públicas

(Partido Nacional, 2019: 115).

Ante esta realidad, se afirma que es preciso probar nuevas estrategias y evolucionar en la manera de abordar lo social. Esto tiene como uno de sus ejes, lo que el documento denomina nuevo concepto de pobreza. Se indica que esta no consiste en la insuficiencia de recursos que permitan atender las necesidades básicas, sino en la incapacidad para autónomamente, generar esos recursos. Esa autonomía se la entiende asociada a empleos dignos y emprendimientos sustentables. En función de esto, la buena política contra la pobreza debe orientarse a crear las oportunidades para que los individuos y las familias, tengan la capacidad de generar ingresos y cooperar con el resto de la sociedad (Partido Nacional, 2019).

Como puede observarse, se trata de una nueva conceptualización de la pobreza que, salvo la mención -y al pasar- a empleos dignos, transcurre por fuera de toda discusión sobre la calidad de los empleos y/o los ingresos. En los apartados en que los documentos se refieren al trabajo, lo hacen básicamente como preocupación por la pérdida de puestos de trabajo. El Partido Nacional (2019), en el punto 'Reactivar la producción y el empleo', plantea seis propuestas; ninguna refiere a la calidad del trabajo o los bajos salarios. Desde este punto, no hay reflexión sobre la pobreza y menos aún, sobre la desigualdad. 
Tampoco aparecen estas cuestiones en el documento de la Coalición Multicolor. En el apartado sobre el 'Impulso al crecimiento: desarrollo productivo y mejora de la competitividad', no hay referencia al trabajo. Se señala que

A la riqueza nacional la generamos los uruguayos con nuestra creatividad, nuestra energía y nuestro esfuerzo. Por eso se necesita reactivar la economía, generando un país más competitivo y dinámico en todos los sectores. Es fundamental implementar medidas que mejoren las condiciones de vida de los uruguayos

(VVAA, 2019).

Y para esto, parece alcanzar la sola existencia de puestos de trabajo.

En el mismo documento, en el punto 'Proteger el mundo del trabajo', hay una mención a los salarios sumergidos, señalando la necesidad de defender su poder de compra. Luego se indica que "Para que la política salarial sea sostenible y el salario real crezca a lo largo del tiempo, se pondrá énfasis en mejorar los niveles de productividad" (VVAA, 2019). En los mismos términos se desarrolló el discurso de Lacalle Pou (2020) al asumir la Presidencia. En estos aspectos, la única preocupación es por el desempleo y a partir de esto, los ejes se centran en los costos de producir, comercializar y prestar servicios, por tanto, el gasto público.

Para generar puestos de trabajo se plantea reducir la carga tributaria. La acción de gobierno se centra allí,

El objetivo será primero desacelerar, y después detener y revertir el crecimiento de la deuda pública, sin aumentar la carga impositiva y tendiendo a mediano plazo hacia una disminución de impuestos. Así lograremos que las personas y las familias dispongan de más recursos y que las empresas sean más competitivas

(Partido Nacional, 2019: 6).

Más adelante se insiste en esta perspectiva, asumiendo que el crecimiento traerá empleo y eso automáticamente, mejorará las condiciones de vida:

La riqueza nacional la generamos día a día los uruguayos con nuestra creatividad, nuestra energía y nuestro esfuerzo. Es por eso que se necesita reactivar la economía, generando un país más competitivo y dinámico en todos los sectores. Además de corregir la situación fiscal, 
es fundamental implementar medidas que promuevan la competitividad en sí misma, es decir, influyendo positivamente en la vida cotidiana de las empresas del país, sea cual sea su tamaño y en más empleo y mejores condiciones de trabajo para todos los uruguayos

(Partido Nacional, 2019: 24).

Esta visión también es la del documento 'Compromiso por el país', haciendo responsable a la política fiscal y cambiaria de los gobierno anteriores de "poner contra las cuerdas" al sector productivo y la consiguiente generación de empleo. La única vinculación desde el trabajo a la pobreza y la asistencia, aparece en el mantenimiento del programa 'Uruguay Trabaja', en el entendido que son los sectores más vulnerables, los que necesitan el mayor apoyo del Estado (VVAA, 2019).

Es también relevante analizar lo que se plantea respecto de las políticas sociales universales (o con esa orientación) en tanto la política asistencial procura básicamente contribuir al acceso y permanencia en las mismas.

En materia de salud se plantea reforzar los acuerdos institucionales y con la comunidad organizada, de manera de apuntalar la coordinación en el primer nivel de atención. Agrega el documento de la Coalición Multicolor que esto se realiza buscando mejorar la eficacia de las acciones orientadas a los grupos de mayor riesgo. Asimismo, el Ministerio de Desarrollo Social aparece mencionado en la puesta en marcha, junto con el de Salud Pública, de un Plan Nacional de Adicciones y Salud Mental (Partido Nacional, 2019;VVAA, 2019). Ninguno de los dos documentos problematiza la desigual calidad de la atención entre el subsistema público y privado. Fundamentalmente, en el caso del Partido Nacional (2019), las preocupaciones por las inequidades regionales están en el interior del país, en particular en el ámbito rural.

El diagnóstico es crítico en educación. La Coalición Multicolor afirma la existencia de una "emergencia educativa"; el Partido Nacional plantea la necesidad de "revertir las tendencias de los últimos años" que han generado "fractura y exclusión". Se denuncian problemas de cobertura, desvinculación, inequidad y calidad del aprendizaje; la situación, mala en la educación básica es aún más grave en la educación media. En particular se destacan como problemas los aprendizajes insuficientes, el abandono antes de cumplir la educación obligatoria y la falta de equidad, en tanto quienes abandonan antes y aprenden menos, son los más pobres. En respuesta a esa realidad el Partido Nacional propone básicamente medidas de carácter institucional y el diseño curricular. En este contexto, una de las propuestas refiere a la articulación de las políticas educativas con las de inclusión. La Coalición Multicolor indica medidas como la extensión del tiempo pedagógico y de permanencia de los alumnos en los centros educativos de educación inicial y primaria y de centros modelo de educación media 
en barrios vulnerables de todo el país, que funcionarán en el marco de una nueva modalidad educativa. También se plantea el desarrollo de proyectos de formación técnico profesional para jóvenes que no estudian ni trabajan, para iniciarlos en el aprendizaje de oficios y en la incorporación de nuevas tecnologías constructivas.

En materia de vivienda el documento de la Coalición Multicolor propone una política activa de regularización de asentamientos, fortalecimiento del programa Movimiento Pro Erradicación de laVivienda Insalubre Rural (MEVIR) y reformulación de la institucionalidad en la materia, promoción de nuevas modalidades de crédito y de sistemas constructivos, de la ayuda mutua y autoconstrucción y la reducción de costos fiscales (VVAA, 2019). El Partido Nacional señala que el país se encuentra en una situación de emergencia en vivienda y que existe un grave déficit habitacional que es una de las causas del aumento del número de asentamientos. Dentro de las orientaciones estratégicas, se indica dar prioridad a los más carenciados y vulnerables y favorecer la integración territorial, para evitar "fragmentaciones generadoras de sub culturas". El MIDES aparece mencionado en el caso de las regularizaciones de asentamientos, actuando de manera conjunta con otros organismos.

Llama la atención la mención relevante a la pobreza, a distancia de otras arenas de política y del mercado de trabajo.También lo hace el que la preocupación por los asentamientos -como expresión de esa realidad- ocurra casi sin reflexión sobre los motivos que los generan. Estos aparecen explicados desde lo realizado por los gobiernos del Frente Amplio:“(...) la ineficacia está claramente vinculada a problemas de gestión e insuficiencia de recursos, además de que en el arsenal de instrumentos que maneja el Ministerio deVivienda, Ordenamiento Territorial y Medio Ambiente (MVOTMA), quedaron fuera los más pobres" (Partido Nacional, 2019: 151). Más allá de la frase indicada en el párrafo anterior, se visualiza también la ausencia de problematización sobre los procesos de segregación territorial, que afectan a distintos sectores de la sociedad uruguaya y -nuevamente- su vinculación con la desigualdad.

Asimismo, se observa que la preocupación por la vivienda, no supone un reposicionamiento del Estado y sus acciones parecen más orientadas a abrir el espacio al sector privado. Una de las orientaciones estratégicas es la promoción y estímulo de la inversión privada y dentro de las medidas se señala bajar de manera rápida el costo de la construcción pública, considerando que construir es caro en comparación con otros países. También se proponen adecuaciones legales y normativas para favorecer el desarrollo de sistemas constructivos no tradicionales y celebrar convenios con organizaciones con experiencia en soluciones habitacionales de urgencia, promoviéndose el involucramiento de los beneficiarios (Partido Nacional, 2019).

En general, no se observa en estos documentos una reflexión en términos de protección social, del papel del Estado en esta y los desafios, límites y posibilidades para articular efectivamente las políticas asistenciales con las restantes políticas sociales y el mundo del trabajo. 
A distancia de preocupaciones sobre los asuntos mencionados, la pobreza tiene en la esperanza su eje central:

Luego de años en los que primó una concepción asistencialista y tutorial, nosotros afirmamos que la única herramienta eficaz para superar la exclusión y la marginalidad es la esperanza. ${ }^{8}$ Una esperanza que sea compartida por la sociedad en su conjunto, por los beneficiarios de las políticas públicas y por quienes las aplican. El convencimiento de que la exclusión y la pobreza pueden ser derrotadas es la base del desarrollo social (Partido Nacional, 2019: 115).

La esperanza no figura en el documento de la Coalición Multicolor pero es un eje clave en la orientación del MIDES. En una intervención periodística el ministro Bartol afirma que es el motor de salida de las situaciones de pobreza, entendiendo que la desesperanza (que ocurre cuando una persona imagina el futuro peor que el presente) genera un obstáculo en la cabeza que no hace ver la solución, “que a veces, está ahí". Asimismo, responde a quienes critican esa centralidad de la esperanza. Considera que se la visualiza de una manera muy liviana, sin entender el trasfondo que está a lo largo del programa y que supone confiar en cada una de las personas y que todas tienen capacidad para salir adelante, especialmente cuando alguien está al lado y empuja a salir. Agrega que cuando la gente está en esa situación de gran depresión, no se la soluciona con técnicos. Se arregla con gente hablándole a la par y dándole ánimo. ${ }^{9}$

La nueva mirada supone centralmente dos aspectos: cambios en el MIDES y en las políticas sociales. El primero de estos aspectos es parte de ambos documentos. En el elaborado por la Coalición Multicolor, como primera medida en la protección a los más débiles se plantea: "Reorganizar y redefinir las estrategias de acción del MIDES, incrementando su efectividad, reduciendo la burocracia y la discrecionalidad, descentralizando la ejecución de los programas sociales y coordinando acciones con otras dependencias del Estado, con los gobiernos departamentales, con las familias y la sociedad civil" (VVAA 2019). Mayor desarrollo y posiblemente radicalidad se observa en el programa del Partido Nacional. Allí se habla del fracaso del MIDES y esto aparece también asociado a discrecionalidad y burocracia. Esta nunca es entendida como parte necesaria de la institucionalidad estatal y es siempre visualizada como perjudicial. De hecho, la primera de las acciones a impulsar es también la reorganización de este organismo, comenzando con desmontar el formato burocrático (Partido Nacional, 2019).

Otro aspecto a destacar refiere a los recursos y esto supone dos aspectos. Por una parte, no se ubica allí el problema, se entiende que han sido suficientes y en cambio se reitera la 
idea de esfuerzo ya hecho por la población uruguaya. Se trata de una afirmación que no dialoga con la distribución del gasto público (en particular los montos de la política asistencial), ni con las condiciones de vida de las personas a las que esta se dirige. Se señala que

El problema no ha sido la falta de recursos, sino la manera en la que se utilizaron. Con el paso de los años, el MIDES se convirtió en una macroestructura ineficiente $y$ politizada, rodeada de una red de operadores que ganan dinero con sus intervenciones $y$, por lo tanto, terminan convirtiéndose en socios de la pobreza. (...) En buena medida, los recursos que la sociedad uruguaya aporta con esfuerzo para combatir la exclusión han terminado financiando amiguismo y burocracia

(Partido Nacional, 2019: 115).

Esto también hizo parte del discurso del ministro Bartol (2020), indicando que el MIDES tiene un presupuesto importante y que con la misma plata se puede trabajar muchísimo mejor, pudiendo hacer mucha reasignación de fondos para atender prioridades.

La política social no es visualizada como soporte necesario para la construcción del individuo y su orientación general es residual. De acuerdo al documento del Partido Nacional

Si le pidiéramos a la gente que siga haciendo el esfuerzo, sólo agravaríamos los problemas: la actividad económica se vería todavía más frenada y se agudizarían problemas sociales como el desempleo. Por eso decimos que esta vez el esfuerzo tiene que hacerlo el Estado. Proponemos gobernantes responsables y austeros, que tomen decisiones sobre los recursos públicos con el mismo cuidado con el que administran su propio dinero. Proponemos un Estado eficiente, que deje de gastar dinero en programas que nadie evalúa y en la multiplicación de cargos innecesarios. Proponemos la incorporación de tecnología y de nuevos mecanismos de control que permitan hacer un seguimiento día a día de las decisiones. Proponemos políticas públicas más inteligentes y mejor coordinadas, que eviten superposiciones y aseguren un mejor uso de los recursos. Proponemos una cultura de gobierno que deje de festejar el aumento del gasto como un fin en sí mismo, y festeje el logro de mejores resultados a menores costos. La alternativa no es entre el despilfarro y el ajuste. Existe 
otro camino, que consiste en gobernar con responsabilidad para liberar recursos que permitan igualar oportunidades, alentar a los que producen y proteger a los más débiles (Partido Nacional 2019: 4).

Las políticas sociales son visualizadas como un límite a la posibilidad de configurarse como individuo y por tanto la orientación es a su finalización. De hecho, se afirma que es una buena política social, la que se vuelve innecesaria (Partido Nacional, 2019).

Como fuera indicado, la mirada sobre las políticas sociales es básicamente en clave de dependencia -que debe superarse- respecto del Estado. Están orientadas por once principios, siendo uno de ellos el de dignidad y sentido de la vida y allí se afirma que si bien distribuir apoyos materiales y oportunidades, es algo importante, el objetivo final de la política social es construir identidad, conciencia de la dignidad personal y sentido de vida. Se entiende que solo quien ve la vida propia como valiosa, tendrá motivos para aprovechar las oportunidades y recursos a su alcance. Una de las consecuencias de esta perspectiva es que las políticas sociales deben organizarse de un modo que no generen dependencia ni asistencialismo. Se afirma que son un esfuerzo que realiza toda la sociedad para convertir a cada persona en protagonista de su vida, siendo su propósito superar la vulnerabilidad, a partir de crear oportunidades e incentivos que pongan a las personas en condiciones de actuar con autonomía y así construir su propia felicidad (Partido Nacional: 2019: 117).

Otro de los principios que guían las políticas sociales es el de centralidad de las personas, confirmando los aspectos que se vienen indicando

El protagonista de las políticas sociales no puede ser un ministerio, ni un conjunto de programas, ni los técnicos que lo llevan adelante, sino el beneficiario concreto. El desarrollo social debe focalizarse en la dignificación de la persona a través del ejercicio efectivo de los derechos y obligaciones, y del fortalecimiento de capacidades. Sin estrategias de desarrollo personal respetuosas de la diversidad de estilos y preferencias, no hay superación de la exclusión ni de la dependencia. Esta idea debe permear el diseño de las políticas, la asignación de recursos y la gestión. Por ejemplo, hay que avanzar hacia un sistema de información que permita conocer todas las acciones de las que se está beneficiando o se ha beneficiado una persona especifica, las oportunidades de apoyo que están a su disposición y aún no ha utilizado, su recorrido histórico y el grado de cumplimiento de las condicionalidades que eventualmente se le hayan establecido. 
Centrarnos en la persona por encima de los limites burocráticos es una condición para ser más eficientes y eficaces, $y$ también para ser más justos

(Partido Nacional: 2019: 117).

En similar sentido se había expresado el ministro Bartol durante la campaña electoral. De acuerdo a la nota citada más arriba, el lema del Ministerio sería 'El MIDES sos vos'. Y agregó: 'No mires para el costado, no busques que alguien resuelva los problemas. En primer lugar, en tu barrio, en tu contexto, el MIDES sos vos y te vamos a respaldar. Te vamos a dar capacitación y a poner un técnico que esté a la orden tuya. Te vamos a reunir con otros que hacen esa misma tarea en otros barrios para que intercambies experiencias. El centro va a ser que vamos a ayudar a los que ayudan'.

La dimensión territorial es otro de los ejes orientadores, indicando que en la formulación e implementación, el territorio es soporte fisico y además, escenario y condicionante. Esto supone que deben reconocerse las diferentes realidades socio culturales y geográficas del país, en tanto implican variantes en el marco institucional (esto es, de niveles de gobierno, entes y sectoriales), en los actores y recursos involucrados. Esto tendrá impactos en la potencia de la intervención del Estado (Partido Nacional, 2019).

La referencia al territorio ocurre sin considerar los procesos de segregación territorial instalados en el país; apuesta a movilizar recursos locales que el documento denomina 'redes comunitarias':

Las politicas que impulsamos proponen fortalecer a los líderes locales y a las redes comunitarias como agentes de cambio. Queremos colocar a la comunidad como protagonista del proceso de salida de la exclusión. El empoderamiento de la comunidad es una alternativa a la cultura de clientelismo asistencial, que genera lazos de dependencia entre los beneficiarios y la burocracia estatal. Nosotros apostamos a una acción pública que movilice la energía de la comunidad. Esto requiere la construcción de lazos personalizados entre referentes locales, el personal del Estado y cada uno de los beneficiarios, en un proceso que parte de un excluido y culmina en un actor social activo e integrado. Para esto es vital encontrar los liderazgos y movilizar las capacidades y competencias que existen en el territorio. También implica aprovechar el conocimiento diseminado en las propias comunidades. Nadie conoce mejor las particularidades de cada situación, las urgencias 
y las oportunidades que las personas y organizaciones activas a nivel local

(Partido Nacional: 2019: 117-118).

A lo señalado, se agrega el denominado modelo de proximidad. Hay en él una crítica a los procesos de estandarización del ingreso a la política asistencial pero la respuesta resulta preocupante:

No es posible interpretar la realidad a partir de un formulario, ni evaluar la pertinencia de una prestación mediante un algoritmo. Debemos estar presentes en el territorio, para que el Estado se transforme en apoyo para la superación de cada persona y del colectivo que integra. Nos proponemos trabajar con una red de mesas territoriales conformadas por referentes locales que, actuando colectivamente, sean los verdaderos protagonistas en la distribución de los recursos, ya que su conocimiento de la realidad les permite comprender las situaciones de mayor urgencia y necesidad. El principal papel de los técnicos será aportar su conocimiento sobre las fuentes de recursos disponibles, apoyar en materia de capacitación y buenas prácticas, realizar el seguimiento y evaluar resultados. También serán claves en el apoyo de las mesas territoriales para su normal funcionamiento (Partido Nacional: 2019: 118).

De acuerdo al ministro Bartol

Lo que tenemos que coordinar es a la gente. Si a ellos mismos les das capacidades para que se organicen entre sí, van a ir saliendo adelante'. [El MIDES debe] acercarse a la gente como uno más. No como un técnico o burócrata. Que no venga alguien de otro contexto muy distinto al mío a hablarme de cosas que nunca vivieron. Para que esa persona salga, primero se tiene que convencer de que puede salir.' ${ }^{10}$

En consonancia con lo anterior este modelo toma como una de sus expresiones a la figura del mentor, operando junto con las organizaciones de la sociedad civil:

El proceso de superación del individuo se ve fortalecido si es acompañado por quienes ya han transitado ese camino, conocen las dificultades y son el ejemplo tangible de que es 
posible salir adelante. Esta tarea estará a cargo de mentores debidamente seleccionados, capacitados y orientados (Partido Nacional, 2019: 118).

En ambos documentos destaca el papel de la familia. Afirman que las personas no viven solas sino en comunidades donde se desarrollan y crean vínculos y la primera de ellas, y fundamental, es la familia. Las políticas sociales no deben centrarse en los individuos sino en los entornos familiares, articular con ellos y preservarlos; la familia es el agente fundamental de protección, contención e impulso al desarrollo personal. Las políticas deben dar prioridad a la primera infancia y asegurar atención integral a todos los niños. Se propone en ambos mejorar la eficacia de las asignaciones familiares y otras formas de transferencias monetarias. La legitimidad de estas acciones no deriva del derecho de las personas a una vida digna y al acceso a recursos sino a una adecuada relación costo/ beneficio del gasto social:

(...) está demostrado que el dinero invertido en los primeros años es el que tiene un mayor impacto sobre la vida de los beneficiarios y sobre la sociedad en su conjunto. No existe otra política que sea a la vez tan justa y tan redituable para la sociedad. El gasto público en la primera infancia no plantea ninguna disyuntiva entre eficiencia y equidad. Mejorar las condiciones de vida de nuestros niños mejorará en el mediano y largo plazo la educación, la economía, la seguridad y el funcionamiento de las instituciones políticas. A diferencia de la mayoría de las otras inversiones, la inversión en educación infantil aborda la desigualdad al mismo tiempo que promueve la productividad económica y mejora la convivencia social (Partido Nacional, 2020: 120).

Asimismo, se postula reformular y fortalecer las asignaciones familiares y vincularlas a las que contempla el Plan de Equidad, de manera de alentar la búsqueda de empleo y reconocer el esfuerzo contributivo del trabajador formal (Partido Nacional, 2020).

Finalmente, también se realizan indicaciones sobre las formas de gestión de las políticas sociales y también aquí se observa el carácter residual del papel del Estado. Se indica que es preciso distinguir entre la rectoría y la ejecución de las políticas sociales. Es tarea del MIDES fijar objetivos, definir estrategias, asignar recursos y evaluar impactos y proponer acciones de mejora. Pero la guía de trabajo será el principio de subsidiariedad (que supone que la intervención pública se vuelve más sensible y eficiente cuando es desarrollada por quien está más cerca de los interesados), la descentralización de las políticas 
y la gestión local. Esto hace que la ejecución de los programas, deba realizarse junto con los gobiernos departamentales, los municipios, la sociedad civil y las organizaciones voluntarias. Esto supone sustituir lo que se entiende como modelo de gestión burocrático, por uno nuevo, que el documento denomina de proximidad (Partido Nacional: 2019).

Además, serán integradas organizaciones sin fines de lucro, que solo recibirán dinero público para el cumplimiento de metas que serán adecuadamente evaluadas.

\section{Síntesis final y conclusiones}

Durante la campaña electoral de 2019, uno de los temas estuvo centrado en la pobreza, las respuestas a la misma y la institucionalidad básicamente encargada de su gestión. Se trata de asuntos, acciones e instituciones que fueron un eje relevante en los gobiernos progresistas. El Partido Nacional previamente a las elecciones expresó que algunos Ministerios no serían negociables, siendo uno de ellos el de Desarrollo Social, cuyas tres máximas autoridades -y a diferencia del resto de los Ministerios- están a cargo de hombres de confianza del Presidente Lacalle Pou.

En base a las propuestas de gobierno elaboradas por el Partido Nacional y la coalición multicolor y a declaraciones del Presidente y Ministro de Desarrollo Social, este artículo tuvo como objetivo presentar y problematizar las orientaciones en relación a la política asistencial. Se trata de orientaciones también puestas en evidencia en la Ley de Presupuesto y en las políticas implementadas en el contexto de la pandemia.

Se plantea una nueva concepción de la pobreza, que, a cuenta de lo complejo, simplifica. Se reitera la referencia a oportunidades que deben aprovechar quienes viven en esas condiciones. Además, indicando que la pobreza no es solo la suficiencia de recursos, se obvia la discusión sobre los mismos y las oportunidades existentes y su distribución. Se trata de una reflexión sobre la pobreza que opera por fuera de toda consideración sobre la desigualdad. Tal como señala Merklen (2013), las políticas del individuo toman como población a los perdedores por haber perdido, pero no se ocupan de las circunstancias que llevan a unos a ganar y a otros a perder.

Las propuestas analizadas no realizan consideraciones sobre la pobreza y menos aún respecto de la desigualdad social. La sola preocupación es el desempleo, sin ingresar en la calidad de los empleos generados. Se alienta la idea que el simple trabajo genera la salida de la pobreza, sin discutir ni problematizar la enorme cantidad de trabajadores y trabajadoras pobres que existen en América Latina. Asimismo, se lo hace de la idea que el crecimiento económico genera automáticamente mejoras en el bienestar social. La vieja idea de primero 'hacer crecer la torta', sin nunca discutir los diversos tamaños de sus pedazos, vuelve a ser planteada. El Estado aparece asociado a una pesada carga que 
limita y dificulta a los emprendedores, que son quienes se entiende generarán trabajo y por tanto oportunidades.

Se trata de valoraciones que se realizan en mercados de trabajo donde -y aún a pesar de las mejoras operadas a partir de los años 2000- un número importante de trabajadores y trabajadoras, sobrevive con niveles salariales muy por debajo de condiciones de subsistencia dignas y decentes y persisten altas tasas de informalidad y precariedad laboral de manera especial entre los trabajadores menos calificados y que pertenecen a los deciles más bajos. Asimismo, se mantienen las brechas entre hombres y mujeres en los salarios y en el acceso a cargos de responsabilidad. Finalmente, la tasa de desempleo es superior entre los jóvenes y dentro de esto, destaca la situación de las mujeres jóvenes, y esto a pesar del aumento del nivel educativo medio de los trabajadores (Olesker 2016).

Son estas tendencias confirmadas en el momento de elaborar las propuestas de gobierno. De acuerdo al informe de la Organización Internacional del Trabajo (OIT) de 2019, se observa un proceso de desaceleración económica desde mitad del año 2018, con impactos en la estructura y calidad de los empleos en el continente. Destaca el documento que se mantiene la tendencia al menor crecimiento del empleo asalariado en relación al cuenta propia, indicando un aumento de la informalidad. Agrega la desaceleración del crecimiento del empleo registrado en 2019, junto con el aumento de la subocupación por insuficiencia de horas.

El eje de la mirada de la pobreza es la 'incapacidad' de los pobres para generar ingresos en forma autónoma, hay una apuesta a la dignidad, al protagonismo y a un proceso interno de transformación, que -nuevamente- se propone por fuera de la consideración de las condiciones objetivas que lo limitan o hacen posible. La acción del Estado, parece casi exclusivamente centrada en estimular estos procesos. Las referencias a la acción del MIDES son básicamente las de inspirar a cambiar, trasmitir confianza, hablar y dar ánimo. Dirá Merklen (2013) que las políticas del individuo apuestan a este en tanto tal y tienen su producción como objetivo. Requerirán que sea responsable y que se mantenga activo. Suponen en esencia, un trabajo de subjetivación y así, se completa una redefinición de las causas, efectos y posibles soluciones a los problemas sociales.

Igual que en la década de 1990, se observa la desvalorización del factor económico en la conceptualización de la pobreza y las respuestas a la misma. Como señalan Lo Vuolo et al (1999) esto puede llevar a sostener que no es en la política económica donde hay que buscar las salidas a la pobreza sino en una mejor organización comunitaria, la ausencia de canales de participación, la falta de motivación para concurrir a la escuela. Se revierten las relaciones causales y no sería la falta de ingresos la que limita las posibilidades de educación, sino la falta de esta la que limita acceder a mejores ingresos. De esta forma, la solución pasa por los propios pobres. 
Hay además una apuesta a los pares y a los contextos barriales, siendo uno de los ejes del modelo de políticas sociales propuesto por el Partido Nacional. Se trata de una apuesta que no es discutida desde los procesos de segregación territorial, ni desde los recursos que pueden ser movilizados por familias y comunidades pobres. Esto implica un retorno a la individualización y la comunitarización planteada por Danani (2009) como características de los años 90. Suponen para la autora la pérdida del carácter social y colectivo de la vida. Comparten la negación -en sentido material, institucional y político-cultural- por la responsabilidad social por el bienestar. Tampoco se lo hace desde procesos ocurridos en el contexto de descentralización, en relación a los cuales Melo (1997) destaca la clientelización en los programas sociales. Observa porosidad del gobierno local en relación a las élites locales, lo que hace que los riesgos de captura del Estado, por estas, sean muy grandes, además de la clientelización de las condiciones de supervivencia de sectores vulnerables.

Esta preocupante perspectiva se vuelve aún más compleja cuando se la ubica en una mirada general sobre el bienestar y la protección social. Como fuera analizado, los diversos regímenes de bienestar, suponen presencia estatal, pero de características distintas. Las críticas a los resultados alcanzados por los gobiernos del Frente Amplio, no redundan en una defensa de la orientación universal del Estado. Al contrario, es colocado en un lugar residual, no siendo considerado como un pilar central en el acceso al bienestar. Las referencias son a un mayor apoyo a los sectores vulnerables y a generar mejores condiciones para la acción de los privados. A tono con la perspectiva de Merklen (2013) presentada, las políticas sociales aparecen asociadas a la dependencia y la autonomía es entendida por fuera de la acción estatal.

Además, dentro de esta mirada negativa a los gobiernos precedentes, en la reestructura del MIDES se colocan parte importante de las apuestas. La acción de ese organismo -y en general la del Estado- es siempre entendida como burocrática y esto connotado de manera exclusivamente negativa. Cuesta entender como se considera posible institucionalizar políticas y programas, por fuera de la burocracia. Además de lo indicado sobre los procesos de clientelización, tampoco es claro como se jerarquizarán las demandas y necesidades identificadas por los diversos referentes barriales.Y tampoco lo es, el diálogo entre ese proceso y la definición de estándares definidos como socialmente deseables para el conjunto de las personas que habitan el país.

Las intenciones son muchas veces insuficientes, pero siempre importantes. Las que pueden observarse en el material analizado muestran una nueva versión de los años 90, lo que presumiblemente traerá las mismas consecuencias. Aunque en un tiempo nuevo, es esperable la agudización de dramáticos trazos de larga data. 
${ }^{1}$ Los otros ministerios son el de Economía y Finanzas y el de Educación y Cultura: www.telenoche.com.uy/ nacionales/lacalle-pou-confirmo-tres-candidatos-ministros

${ }^{2}$ ladiaria.com.uy/politica/articulo/2019/11/cabildo-abierto-y-el-partido-colorado-pujan-por-el-ministerio-de-vivienda

${ }^{3}$ Las comillas son del autor. Esping-Andersen (2000) reconoce asimismo un papel importante a las asociaciones voluntarias y sin fines de lucro.

${ }^{4}$ Creado por ley 17.869, se integra por los siguientes componentes: Plan Alimentario Nacional; Programa Emergencia Sanitaria; Programa de Ingreso Ciudadano; Programa Educación en Contextos Críticos; Programa Empleo Transitorio; Programa Asentamientos Precarios y Pensiones y Programa Alojamiento a las Personas en Situación de Calle (Baráibar 2018).

${ }^{5}$ Forman parte de este componente un conjunto de reformas en las cuales ya venía actuando el gobierno del Frente Amplio: la reforma tributaria; la política de salud; las políticas de empleo; la política de vivienda; la política educativa y el Plan de igualdad de oportunidades y derechos (CNPS, 2008).

6 https://www.elobservador.com.uy/nota/lacalle-asegura-reestructura-del-mides-con-jurista-de-su-confianza-20191213101616

${ }^{7}$ www.elobservador.com.uy/nota/lacalle-pou-soy-liberal-creo-en-la-libertad-de-las-personas--2020124141348

${ }^{8}$ Énfasis en el documento original.

${ }^{9}$ https://ladiaria.com.uy/politica/articulo/2019/10/pablo-bartol-el-lema-del-ministerio-sera-el-mides-sos-vos/

${ }^{10} \mathrm{https}$ ///www.elpais.com.uy/informacion/politica/pablo-bartol-figura-lacalle-pou-apuesta-levantar-mides.html 


\section{Referencias bibliográficas}

Andrenacci, Luciano y Repetto, Fabián (2006) "Un camino para reducir la desigualdad y construir ciudadanía”. En Carlos Gerardo Molina (ed.) Universalismo básico. Una nueva política social para América Latina. Washington D.C.: BID, 2006: 83-111 Disponible en: www.publications.iadb.org

Arias, Ana Josefina (2012) Pobreza y modelos de intervención. Aportes para la superación del modelo de asistencia y promoción. Buenos Aires: Espacio Editorial.

Baráibar, Ximena (2018) "La política asistencial en el Uruguay progresista: trazos y tensiones", en Andrenacci, Luciano; Campana, Melisa y Servio, Mariana (coords): La asistencia social en Argentina y América Latina: avances, problemas y desafíos. PEGUES. Rosario. Disponible en: https://issuu.com/pegues/docs/asal_2019

Barba Solano, Carlos (2018) "Liberalismo y universalismo en tensión: 25 años de reformas sociales en los regímenes de bienestar en América Latina”. En Midaglia, Carmen; Ordóñez, Gerardo y Valencia, Enrique (coord.) Políticas Sociales en América Latina en los inicios del siglo XXI. Innovaciones, inercias y retrocesos. Buenos Aires: CLACSO/ El Colegio de la Frontera Norte: 75-105.

Barba Solano, Carlos (2019) "Desigualdad y regímenes de bienestar. Una mirada teórica”. En Bayón, María Cristina (coord.) Las grietas del neoliberalismo. Dimensiones de la desigualdad contemporánea en México. Ciudad de México: UNAM:145-194.

Boschetti, Ivanete (2001) Assistencia Social no Brasil: um direito entre originalidade e conservadorismo. Brasilia: Universidad de Brasilia.

Campana, Melisa (2014) "Desarrollo humano, producción social de la pobreza y gobierno de la pobreza”. En Trabajo Social, 16, 79-89.

Cecchini, Simone y Martínez, Rodrigo (2011) Protección social inclusiva en América Latina. Una mirada integral, un enfoque de derechos. Santiago: CEPAL/ Giz.

Danani, Claudia (2009) "La gestión de la política social: un intento de aportar a su problematización” En Chiara, Magdalena; Di Virgilio, María Mercedes (org) Gestión de la política social. Conceptos y herramientas. Buenos Aires: UNGS - Prometeo libros, 2009: 25-51.

Esping-Andersen, Gosta (2000) Fundamentos sociales de las economías postindustriales. Barcelona: Editorial Ariel. 
Grassi, Estela (2018 a) "Estado social y desbloqueo de la sociedad neoliberal". En Grassi, Estela y Hintze, Susana (coord.) Tramas de la desigualdad. Las políticas y el bienestar en disputa. Buenos Aires: Prometeo libros: 57-121.

Grassi, Estela (2018 b) “Transformaciones del trabajo y de la política social”. En Grassi, Estela y Hintze, Susana (coord.) Tramas de la desigualdad. Las políticas y el bienestar en disputa. Buenos Aires: Prometeo libros: 123-189.

Lo Vuolo, Rubén; Barbeito, Alberto; Pautassi, Laura y Rodríguez, Corina (1999) La pobreza... de la política contra la pobreza. Buenos Aires: Miño y Dávila Editores/ CIEPP.

Martínez Franzoni, Juliana (2008) Domesticar la incertidumbre en América Latina. Mercado laboral, política social y familias. San José: Editorial Universidad de Costa Rica.

Martínez Franzoni, Juliana y Sánchez-Ancochea, Diego (2016) “Regímenes de bienestar en América Latina: tensiones entre universalización y segmentación”. En del Pino, Eloisa y Rubio Lara, María Josefa (dir.) Los Estados de Bienestar en la encrucijada. Políticas sociales en perspectiva comparada. Madrid:Tecnos, 200-220.

Melo, Marcus André (1997) "Federalismo e política social: as vicisitudes da descentralizacao. En Filgueira, Carlos; Midaglia, Carmen y Petersen-Thumser, Jens (comp.) Desafíos de la seguridad social. Montevideo: Editorial Trilce: 265-288.

Merklen, Denise (2013) “Las dinámicas contemporáneas de la individuación”. En Castel, Robert, Kessler, Gabriel, Merklen, Denis y Murard, Numa: Individuación, precariedad, inseguridad ¿desinstitucionalización del presente?. Buenos Aires: Editorial Paidós, 45-86.

Midaglia, Carmen y Antía, Florencia (2011) “El sistema de bienestar uruguayo: entre la moderación reformista liberal y la ampliación de la responsabilidad estatal en el área de la protección social”. En Idiart, Alma (ed.) Estado benefactor y políticas sociales. Buenos Aires: Editorial Biblos, 21-48.

Midaglia, Carmen y Castillo, Marcelo (2018) “América Latina en el siglo XXI: avances y retrocesos en las pautas de desarrollo social”. Cadernos no CEAS, 245: 660-687.

Midaglia, Carmen y Silveira, Milton (2011) "Políticas sociales para enfrentar los desafios de la cohesión social: los nuevos Programas de Transferencias Condicionadas de Renta en Uruguay”. En Barba Solano, Carlos y Cohen, Néstor (coord.) Perspectivas críticas sobre la cohesión social. Desigualdad y tentativas fallidas de integración social en América Latina. Buenos Aires: CLACSO: 225-249 
Olesker, Daniel (2016) El mercado laboral en América Latina: pasado, presente y futuro. Nueva Sociedad, 264: 63-71.

Queirolo, Rosario (2020) ¿Qué significa el 'giro a la derecha' uruguayo?. Nueva Sociedad, 287: 98-107.

Rosenblatt, Fernando; Piñeiro, Rafael y Pérez, Verónica (2019) ¿Qué hará la derecha uruguaya?. Nueva Sociedad. Disponible en: www.nuso.orgVisitado el 22 setiembre 2020.

Soldano, Daniela y Andrenacci, Luciano (2005) “Aproximación a las teorías de la política social a partir del caso argentino”. En Andrenacci, Luciano (comp.) Problemas de política social en la Argentina contemporánea. Buenos Aires: UNGS - Prometeo libros, 2005: 17-79

Ubasart-González, Gemma y Minteguiaga, Analía (2017) “Esping-Andersen en América Latina. El estudio de los regímenes de bienestar”. Revista Política y gobierno, vol. XXIV (1), 213-236.

\section{Fuentes}

Bartol, Pablo (2020) Discurso pronunciado al asumir como Ministro del MIDES. Disponible en: www.presidencia.gub.uy/comunicacion/comunicacionnoticias/pablobartol-asumio-como-ministro-en-desarrollo-social Fecha de acceso: 28 setiembre 2020.

CNPS - Consejo Nacional de Políticas Sociales (2008) Plan de Equidad, Montevideo: IMPO.

CNPS - Consejo Nacional de Políticas Sociales (2011) La Reforma Social. Hacia una nueva matriz de protección social del Uruguay, MIDES: Montevideo.

Corte Electoral (2019) Elecciones nacionales 2019. Disponible en: www.corteelectoral. gub.uy Fecha de acceso: 20 abril 2020.

Lacalle Pou, Luis (2020) Discurso pronunciado ante la Asamblea General. Disponible en: https://www.presidencia.gub.uy/sala-de-medios/videos/lacalle-pou-discurso-fragmento-asamblea Fecha de acceso: 28 setiembre 2020.

Ley $\mathbf{n}^{\circ} 17866$ (2005) Creación del Ministerio de Desarrollo Social. Montevideo, Registro Nacional de Leyes y Decretos, Disponible en: www.impo.com.uy

Organización Internacional del Trabajo (2019) Panorama Laboral 2019. América Latina y el Caribe. Disponible en: www.oitcinterfor.org 
Partido Nacional (2019) Lo que nos une. Programa de Gobierno 2020/2025. Disponible en: www.partidonacional.org.uy

Presidencia de la República/MIDES (2009) Transición de Gobierno 2009/2010. Disponible en: www.archivo.presidencia.gub.uy

VVAA (2019) Compromiso por el país. Disponible en: www.montevideo.com.uy

\section{Cómo citar este artículo:}

Baráibar Ribero, Ximena (2021) "Lo viejo en lo nuevo: la política asistencial en el Uruguay actual”. Revista Perspectivas de Políticas Públicas Vol. 11 №21: 211-243 